\title{
Clinical Reasoning: Ventriculomegaly detected on 20-week anatomic fetal ultrasound
}

Robert C. Stowe, MD, Ariel M. Lyons-Warren, MD, PhD, and Lisa Emrick, MD

Neurology ${ }^{\circledR}$ 2018;91:e1265-e1268. doi:10.1212/WNL.0000000000006247

\section{Correspondence}

Dr. Stowe

robertcstowe@gmail.com

\section{Section 1}

A 23-year-old G1P0 woman was referred for fetal MRI after her 20-week anatomic fetal ultrasound demonstrated cerebral ventriculomegaly. Her pregnancy to date had been uncomplicated without notable fevers, illnesses, or need for medications aside from prenatal vitamins. The fetal MRI obtained at 24 weeks gestation demonstrated a phenotypically male fetus with severely dilated lateral ventricles measuring up to $30 \mathrm{~mm}$ with marked thinning of the cortical mantle, aqueductal stenosis, diencephalic fusion, and brainstem dysplasia (figure, A and B).

\section{Questions for consideration:}

1. What are the initial diagnostic considerations for a fetus with ventriculomegaly?

2. What recommendations can be made at this time?

3. How would you counsel this mother?

Figure Fetal and postnatal MRI findings
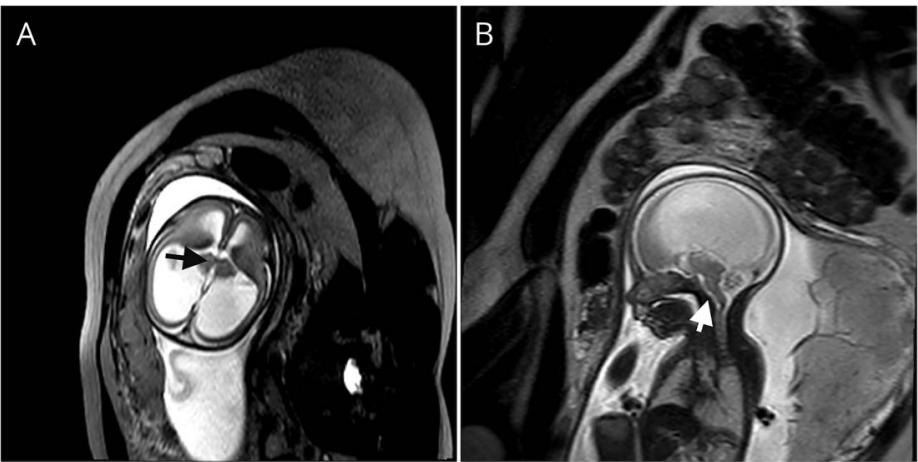

C

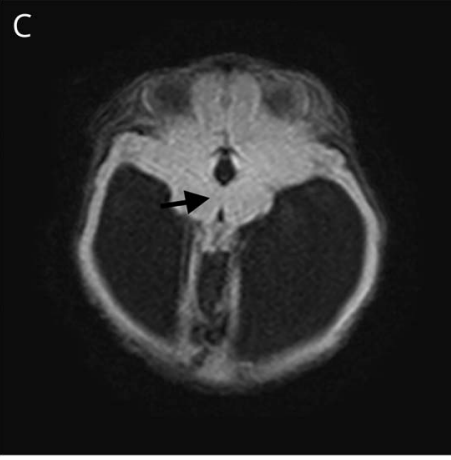

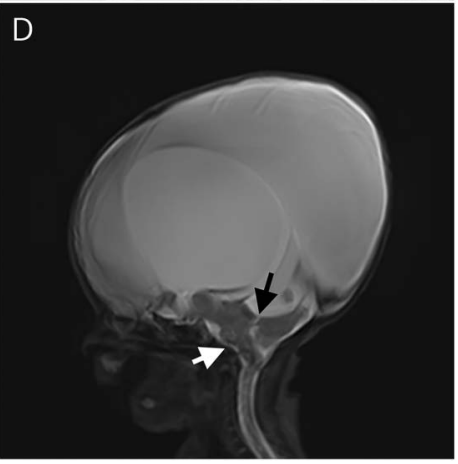

Prenatal T2 axial (A) and coronal (B) MRI show significant hydrocephalus and thin cortical mantle. Diencephalosynapsis (A, black arrow) and abnormally thin and kinked brainstem (B, white arrow) can also be seen. Postnatal fluid-attenuated inversion recovery axia (C) and T2 fast imaging employing steady-state acquisition coronal (D) MRI show significant hydrocephalus with a thin cortical mantle. Aqueductal stenosis (C, black arrow) and abnormally thin and kinked brainstem ( $D$, white arrow) can also be seen.

GO TO SECTION 2

From the Department of Pediatrics, Section of Neurology and Developmental Neuroscience (R.C.S., A.M.L.-W., L.E.), and Department of Molecular and Human Genetics (L.E.), Baylor College of Medicine, Texas Children's Hospital, Houston.

Go to Neurology.org/N for full disclosures. Funding information and disclosures deemed relevant by the authors, if any, are provided at the end of the article. 


\section{Section 2}

The differential diagnosis for fetal ventriculomegaly is broad. ${ }^{1}$ Therefore it is useful to divide fetal ventriculomegaly into isolated ventriculomegaly measuring $10-15 \mathrm{~mm}$ at the level of the atria of the lateral ventricles vs severe causes, which are often associated with a genetic syndrome (table). Isolated, mild ventriculomegaly is usually benign while syndromic ventriculomegaly frequently has associated anomalies and a worse prognosis. Numerous etiologies can produce obstructive hydrocephalus that manifests as fetal ventriculomegaly. Obstructive hydrocephalus without other structural abnormalities suggests blockage along the typical route of cerebrospinal flow, which can be due to hemorrhage, congenital brain tumor, primary brain malformations such as rhombencephalosynapsis, or an isolated malformation such as aqueductal stenosis or secondary obstruction from a Chiari II malformation. Ventriculomegaly can also be a sign of intrauterine infection due to secondary white matter gliosis obliterating the cerebral aqueduct and causing obstruction. ${ }^{1}$ The typical infections to consider are toxoplasmosis and cytomegalovirus, but Zika virus has introduced itself as a new entity that prompts careful travel and exposure histories. ${ }^{2}$ Complex vascular lesions such as vein of Galen malformation can also cause fetal ventriculomegaly. ${ }^{1}$

Recommendations for patients with fetal ventriculomegaly include continued prenatal monitoring of head circumference and body size. In patients with hydrocephalus or a dysplastic brainstem, there can be increased risk of polyhydramnios if the fetus stops effectively swallowing amniotic fluid. ${ }^{3}$ Therefore amniotic fluid status should also be monitored. Delivery should ideally occur at a facility with a neonatal intensive care unit and availability of a pediatric neurosurgery service for moderate to severe causes of ventriculomegaly.
Counseling based on a fetal MRI is challenging due to the varied outcomes. Isolated mild bilateral ventriculomegaly (less than $15 \mathrm{~mm}$ ) demonstrates a prevalence of neurodevelopmental delay of $7.9 \%$, similar to that of the general population. ${ }^{4}$ A favorable outcome may further be expected if no additional cerebral or genetic abnormalities are found postnatally. However, asymmetric, unilateral, or severe ventriculomegaly (more than $15 \mathrm{~mm}$ ) tend to be associated with worse outcomes including high perinatal and neonatal mortality as well as higher frequency of aneuploidy and associated CNS and non-CNS malformations. ${ }^{1}$ This family was counseled that the patient would likely have moderate to severe neurodevelopmental delays and was at risk for postnatal feeding and breathing difficulties.

At 33 weeks and 4 days gestation, the mother delivered via cesarean section following premature rupture of membranes. Initial APGARs of the neonate were 8 and 9 at 1 and 5 minutes, respectively. Examination was notable for significant macrocephaly with fronto-orbital circumference $41.5 \mathrm{~cm}$ (>99 percentile), distended scalp veins, nondysmorphic facies, and fisted thumbs with an otherwise normal general examination. His neurologic examination was unremarkable other than significant head lag attributed to his excessively discordant head size. His tone was otherwise within normal limits for a 33-week premature neonate. A postnatal head ultrasound showed severe bilateral lateral ventriculomegaly measuring up to $86 \mathrm{~mm}$ in maximum craniocaudal dimension and a nondilated fourth ventricle.

\section{Question for consideration:}

1. What further diagnostic workup is warranted at this time?

Table Genetic causes of fetal ventriculomegaly

\begin{tabular}{|c|c|}
\hline Molecular or general class & Syndrome names and associated genes \\
\hline Aneuploidies & Trisomy 13 , trisomy 18 \\
\hline \multirow[t]{3}{*}{$\begin{array}{l}\text { a-Dystroglycanopathies (congenital } \\
\text { muscular dystrophies) }\end{array}$} & $\begin{array}{l}\text { Walker-Warburg syndrome (POMT1, POMT2, FKRP, Fukutin, ISPD, CTDC2, TMEM5, POMGNT1, B3GALNT2, GMPPB, } \\
\text { B3GNT1) }\end{array}$ \\
\hline & Muscle-eye-brain disease (POMGNT1, FKRP, Fukutin, ISPD, TMEM5) \\
\hline & Fukuyama congenital muscular dystrophy (Fukutin, FKRP) \\
\hline Tubulinopathies & TUBA1A, TUBA8, TUBB2B, TUBB3, TUBB5, TUBG1 \\
\hline \multirow[t]{2}{*}{ Ciliopathies } & Meckel-Gruber syndrome (MKS1, TMEM216, TMEM67, TMEM107, TTC21B, TMEM107, CC2D2A, CEP290, RPGRIP1L, TCTN2) \\
\hline & Acrocallosal syndrome (KIF7, GLI3) \\
\hline Congenital disorders of glycosylation & $\mathrm{N}$ - and O-linked disorders; PMM2 is most common \\
\hline X-linked hydrocephalus & L1CAM, AP1S2 \\
\hline Congenital hydrocephalus & EML1, MPDZ, Alkura-Raymond syndrome (KIAA1109) \\
\hline Other metabolic disorders & Pyruvate dehydrogenase complex deficiency, Smith-Lemli-Opitz syndrome, mucopolysaccharidoses \\
\hline
\end{tabular}




\section{Section 3}

Advanced postnatal imaging with MRI may be considered, particularly if neurosurgical planning of ventriculoperitoneal shunting or evaluation for other cortical migrational anomalies is warranted, as in our patient. It may not be necessary if fetal MRI is obtained late in gestation. Postnatal brain MRI in our patient recapitulated severe obstructive hydrocephalus with diffuse stenosis of the cerebral aqueduct associated with a dysplastic, thickened midbrain and tectum, partial midline fusion of dysplastic thalami, and hypoplasia of brainstem with subtle kinking at the pontomesencephalic junction (figure, $\mathrm{C}$ and D). Frontal cortical mantle width was approximately $6 \mathrm{~mm}$.

An ophthalmologic evaluation is important to fully describe the phenotype when considering congenital muscular dystrophies such as muscle-eye-brain spectrum disorders. Similarly, a creatine kinase (CK) level can help rule in or out congenital muscular dystrophies. Testing for congenital disorders of glycosylation (CDG) with transferrin isoelectric focusing is also valuable as these patients can present with mild to severe brain malformations. In the setting of an abnormal diencephalon, evaluation for endocrine abnormalities is worthwhile. Ultimately, genetic testing is exceptionally valuable with comprehensive chromosomal microarray (CMA) and trio whole exome sequencing (WES), ${ }^{5}$ particularly as these multiple brain abnormalities are not classically seen in conjunction with a single isolated syndrome.

In our patient, ophthalmologic examination, CK, CDG testing, thyroid-stimulating hormone, free $\mathrm{T} 4$, cortisol, growth hormone, and CMA were all normal. Trio WES demonstrated a de novo likely pathogenic variant $(c .1703+5 G>A)$ in the L1CAM gene on chromosome $\mathrm{Xq} 28$, previously unreported in the literature. Because the variant occurred within an intronic splicing site, it was predicted to be deleterious.

The neonate was initially clinically stable; however, in the second week of life, he developed frequent apneas and bradycardias, prompting placement of a ventriculoperitoneal shunt. Postoperative imaging did not demonstrate improved cortical mantle width. An EEG was also obtained to determine if there was any epileptic origin to the frequent apneas and bradycardias. The EEG showed excessive discontinuity, multifocal sharp transients, and a depression of the background activity over the posterior regions, but no epileptiform features and no electrographic correlate to his apneas or bradycardias. The patient was discharged home 6.5 weeks after birth on room air, tolerating full enteral feeds, and with multiple subspecialist follow-up appointments. The patient was evaluated at 4 months of age (approximately 2 months corrected gestational age) by neurology and noted to be making good developmental progress by cooing, laughing, reaching for objects, and lifting his head while prone.

\section{Discussion}

The differential diagnosis of fetal ventriculomegaly is broad. As previously described, multiple genetic disorders (table) may contribute to fetal ventriculomegaly. Other fetal developmental abnormalities of the brain, such as agenesis of the corpus callosum, brainstem abnormalities, and cortical migration anomalies, as well as visceral or skeletal anomalies can help narrow the differential diagnosis. As in our patient, many genetic syndromes cannot be differentiated until after delivery. ${ }^{6}$ Abnormal karyotypes are seen in $4.7 \%$ of cases of mild ventriculomegaly. ${ }^{4}$ Karyotypes cannot capture Mendelian disorders or copy number variations and thus the true incidence of genetic disorders in all causes of fetal ventriculomegaly (mild or severe) is unknown.

Based on our patient's hemizygous likely pathogenic variant in L1CAM and his clinical presentation, he was diagnosed with L1 syndrome. The prevalence of L1 syndrome is approximately 1 in 30,000 and the incidence has been estimated at $5 \%$ of boys with nonsyndromic congenital hydrocephalus ${ }^{3}$ although its rarity and broad phenotype make accurate estimations challenging.

Interestingly, the LICAM phenotypic spectrum is broad. Specifically, it includes X-linked hydrocephalus, also known as hydrocephalus due to stenosis of the aqueduct of Sylvius (HSAS syndrome, MIM 307000), in which patients have severe hydrocephalus and adducted thumbs. Another variant is MASA syndrome, in which patients have mild to moderate intellectual disabilities, aphasia, shuffling gait, and adducted thumbs. However, the degree of intellectual impairment does not necessarily correlate with head size or severity of hydrocephalus. X-linked complicated hereditary spastic paraplegia type 1 and X-linked complicated corpus callosum agenesis are 2 other variants. There is a general genotype-phenotype correlation such that patients with aqueductal stenosis and L1CAM truncating mutations have more severe disease and earlier mortality than those with missense mutations. ${ }^{7}$ Intellectual disability tends to be severe when associated with cerebral aqueductal stenosis and not improved with shunting. ${ }^{3}$ For patients with isolated aqueductal stenosis, 2 studies with a total of 92 patients both showed a wide range of neurodevelopmental outcomes from normal to severe disability and a correlation between poor outcomes and low postoperative cortical mantle widths. ${ }^{8}$ However, interpretation of these findings is limited, as comprehensive genetic evaluations were not reported for these patients.

Postnatal imaging findings of neonatal presentations can include hydrocephalus with and without aqueductal stenosis, corpus callosal agenesis or dysgenesis, cerebellar hypoplasia, small brainstem, and agenesis of the corticospinal tracts ${ }^{3}$; no description of diencephalic fusion exists in L1 syndrome. There have been multiple reports of L1CAM pathogenic variants and a risk of Hirschsprung disease; careful monitoring of symptoms of constipation is warranted. ${ }^{9}$ Management of patients with L1CAM pathogenic variants is symptom-based and includes CSF shunting if there is increased intracranial pressure and inclusion of multiple therapy modalities. 
While the diagnosis of L1CAM should be strongly considered in male fetuses with congenital hydrocephalus, many other gene variations can be associated with congenital hydrocephalus, including X-linked AP1S2 and autosomal genes $E M L 1, W D R 81$, and $M P D Z^{10}$ (table), highlighting the utility of trio WES. Genetic diagnosis aids in neurodevelopmental prognostication as well as family planning guidance for recurrence risk in future pregnancies.

\section{Author contributions}

Robert Clinton Stowe: drafting/revising the manuscript, data acquisition, study concept or design, accepts responsibility for conduct of research and final approval. Ariel M. LyonsWarren: drafting/revising the manuscript, data acquisition, accepts responsibility for conduct of research and final approval. Lisa T. Emrick: drafting/revising the manuscript, data acquisition, accepts responsibility for conduct of research and final approval, study supervision.

\section{Study funding}

No targeted funding reported. The work was not sponsored by Baylor College of Medicine, Texas Children's Hospital, or other third parties.

\section{Disclosure}

The authors report no disclosures relevant to the manuscript. Go to Neurology.org/ $\mathrm{N}$ for full disclosures.

\section{References}

1. De Catte L, De Keersmaeker B, Claus F. Prenatal neurologic anomalies: sonographic diagnosis and treatment. Pediatr Drugs 2012;14:143-155.

2. Araujo AQ, Silva MT, Araujo AP. Zika virus-associated neurological disorders: a review. Brain 2016;139:2122-2130.

3. Schrander-Stumpel C, Fryns JP. Congenital hydrocephalus: nosology and guidelines for clinical approach and genetic counselling. Eur J Pediatr 1998;157: 355-362.

4. Pagani G, Thilaganathan B, Prefumo F. Neurodevelopmental outcome in isolated mild fetal ventriculomegaly: systematic review and meta-analysis. Ultrasound Obstet Gynecol 2014;44:254-260.

5. Dyment DA, Sawyer SL, Chardon JW, Boycott KM. Recent advances in the genetic etiology of brain malformations. Curr Neurol Neurosci Rep 2013;13: 364.

6. Amir T, Poretti A, Boltshauser E, Huisman TA. Differential diagnosis of ventriculomegaly and brainstem kinking on fetal MRI. Brain Dev 2016;38: $103-108$.

7. Vos YJ, de Walle HE, Bos KK, et al. Genotype-phenotype correlations in L1 syndrome: a guide for genetic counselling and mutation analysis. J Med Genet 2010;47: 169-175.

8. Villani R, Tomei G, Gaini SM, Grimoldi N, Spagnoli D, Bello L. Long-term outcome in aqueductal stenosis. Childs Nerv Syst 1995;11:180-185.

9. Griseri P, Vos Y, Giorda R, et al. Complex pathogenesis of Hirschsprung's disease in a patient with hydrocephalus, vesico-ureteral reflux and a balanced translocation $\mathrm{t}(3$; 17)(p12;q11). Eur J Hum Genet 2009;17:483-490.

10. Shaheen R, Sebai MA, Patel N, et al. The genetic landscape of familial congenital hydrocephalus. Ann Neurol 2017;81:890-897. 


\section{Neurology}

Clinical Reasoning: Ventriculomegaly detected on 20-week anatomic fetal ultrasound Robert C. Stowe, Ariel M. Lyons-Warren and Lisa Emrick

Neurology 2018;91;e1265-e1268

DOI 10.1212/WNL.0000000000006247

This information is current as of September 24, 2018

\section{Updated Information \&} Services

References

Subspecialty Collections

Permissions \& Licensing

Reprints including high resolution figures, can be found at: http://n.neurology.org/content/91/13/e1265.full

This article cites 10 articles, 1 of which you can access for free at: http://n.neurology.org/content/91/13/e1265.full\#ref-list-1

This article, along with others on similar topics, appears in the following collection(s):

All Genetics

http://n.neurology.org/cgi/collection/all_genetics

Developmental disorders

http://n.neurology.org/cgi/collection/developmental_disorders

Hydrocephalus

http://n.neurology.org/cgi/collection/hydrocephalus

MRI

http://n.neurology.org/cgi/collection/mri

Information about reproducing this article in parts (figures,tables) or in its entirety can be found online at:

http://www.neurology.org/about/about_the_journal\#permissions

Information about ordering reprints can be found online:

http://n.neurology.org/subscribers/advertise

Neurology ${ }^{\circledR}$ is the official journal of the American Academy of Neurology. Published continuously since 1951, it is now a weekly with 48 issues per year. Copyright () 2018 American Academy of Neurology. All rights reserved. Print ISSN: 0028-3878. Online ISSN: 1526-632X.

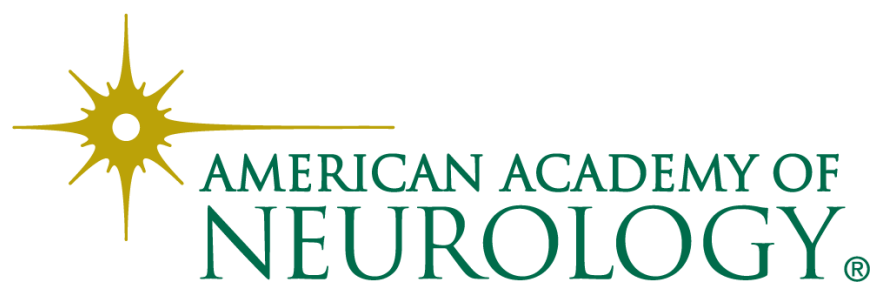

